\title{
sciendo
}

\author{
BULGARIAN ACADEMY OF SCIENCES
}

CYBERNETICS AND INFORMATION TECHNOLOGIES • Volume 21, No 4

Sofia $2021 \quad$ Print ISSN: 1311-9702; Online ISSN: 1314-4081

DOI: $10.2478 /$ cait-2021-0049

\section{A Fuzzy Approach to Multi-Objective Solid Transportation Problem with Mixed Constraints Using Hyperbolic Membership Function}

\author{
Nurdan Kara ${ }^{1}$, Hale Gonce Kocken ${ }^{2}$ \\ ${ }^{1}$ Department of Mathematics, Faculty of Science, Turkish Naval Academy, National Defence University, \\ Turkey \\ ${ }^{2}$ Department of Mathematical Engineering, Faculty of Chemistry-Metallurgy, Yildiz Technical \\ University, Turkey \\ E-mails: nurdancetin@gmail.com hgonce@yildiz.edu.tr
}

\begin{abstract}
Multi-objective Solid Transportation Problem (MSTP) is known as a special class of vector-minimization (or maximization) problems and has three parameters: source, destination, and conveyance. The objectives such as transportation cost, transportation time, transportation safety level, and objectives in terms of environmental and social issues are generally in conflict with each other. In this paper, we present a fuzzy approach to bring these conflicting objectives together as high as possible. Instead of using the linear membership function, which is frequently used in the literature for ease of use, we use the hyperbolic membership function in our approach. Also, while most of the papers in the literature deal with the standard equality constrained form of MSTP, the mixed constrained form is addressed in this paper. Finally, a numerical example from the literature is used to illustrate the construction of the hyperbolic membership function and how well it represents the objective functions' degree of satisfaction.
\end{abstract}

Keywords: Multi-objective optimization, solid transportation problem, hyperbolic membership function, fuzzy mathematical programming.

\section{Introduction}

Solid transportation problem having three items as supply, demand, and conveyance is one of the most important implemented to several real-life problems. If there are multiple non-commensurable and conflicting objectives, then the corresponding problem is called the Multi-objective Solid Transportation Problem (MSTP).

Considering uncertainties in the real world, the fuzzy approach has been studied commonly for multi-objective problems [9] and also for MSTP. B it, B is w al and A l a m [2] have applied the traditional fuzzy approach to solve MSTP. P a m a n i k, $\mathrm{J}$ a n a and Maiti [10] have presented a formulation of MSTP for the damageable item. Chen, Peng and Zhang [6] modeled goal programming for MSTP. 
An uradha et al. [1] have used a row maxima method for solving the bi-objective STP. Leberling [7] solved the multi-objective linear programming problem by fuzzy method with nonlinear membership function. Solution of fuzzy multi-objective problem is found and compared with obtained using trapezoidal and hyperbolic membership functions by Rath and Dash [11, 12]. Peidro and Vasant [8] have implimented the fuzzy goal programming approach with some nonlinear membership functions solving MTPs. The fuzzy approach with hyperbolic membership functions for the multi-objective capacitated transportation problem has been presented by B it [3]. Verma, B is wal and B is was [13] have proposed the fuzzy method using some non-linear membership functions to solve an MTP. B odkhe, B ajaj and Dhaigude [5] have applied the fuzzy approach for solving MSTP using hyperbolic and exponential membership functions. Bit [4] has presented the fuzzy model with hyperbolic membership functions for a multiobjective capacitated STP. The summary of the literature presented is given in Table 1.

Table 1. Summary of the literature presented

\begin{tabular}{|c|c|c|c|}
\hline References & The problem addressed & $\begin{array}{l}\text { Membership } \\
\text { function used }\end{array}$ & $\begin{array}{l}\text { Brief description of } \\
\text { the method used }\end{array}$ \\
\hline $\begin{array}{l}\text { A nur a d h a } \\
\text { et al. [1] }\end{array}$ & $\begin{array}{l}\text { Bi-objective solid } \\
\text { transportation problem }\end{array}$ & Linear & $\begin{array}{l}\text { A row maxima } \\
\text { method }\end{array}$ \\
\hline $\begin{array}{l}\text { B i t, B i s w a l } \\
\text { and A l a m [2] }\end{array}$ & MSTP & Linear & Fuzzy programming \\
\hline B i t [3] & $\begin{array}{l}\text { Linear multiobjective } \\
\text { capacitated transportation } \\
\text { problem }\end{array}$ & Hyperbolic & Fuzzy programming \\
\hline B i t [4] & Capacitated MSTP & Hyperbolic & Fuzzy programming \\
\hline \begin{tabular}{|l|} 
Bodkhe, Bajaj \\
and Dhaigude [5]
\end{tabular} & $\begin{array}{l}\text { MSTP with equality } \\
\text { constraints }\end{array}$ & $\begin{array}{l}\text { Hyperbolic, } \\
\text { exponential }\end{array}$ & Fuzzy programming \\
\hline $\begin{array}{l}\text { Che } n, P \text { e } g \\
\text { and } Z \text { h a n g [6] }\end{array}$ & $\begin{array}{l}\text { Bicriteria solid } \\
\text { transportation problem }\end{array}$ & - & $\begin{array}{l}\text { Expected value and } \\
\text { chance-constrained goal } \\
\text { programming model }\end{array}$ \\
\hline Le berling [7] & $\begin{array}{l}\text { Multi-objective linear } \\
\text { programming problem }\end{array}$ & $\begin{array}{l}\text { Linear, } \\
\text { hyperbolic }\end{array}$ & Fuzzy programming \\
\hline $\begin{array}{l}\text { Peidro and } \\
\text { V a s a n t }[8]\end{array}$ & $\begin{array}{l}\text { Multi-objective linear } \\
\text { transportation problem }\end{array}$ & $\begin{array}{l}\text { Modified S-curve } \\
\text { membership } \\
\text { functions }\end{array}$ & Fuzzy programming \\
\hline $\begin{array}{l}\text { Peneva and } \\
\text { Popchev [9] }\end{array}$ & Multi criteria problems & - & \begin{tabular}{|l|}
$\begin{array}{l}\text { Fuzzy decision } \\
\text { making }\end{array}$ \\
\end{tabular} \\
\hline $\begin{array}{l}\text { Pram a n ik, J a n a } \\
\text { and Mait i [10] }\end{array}$ & $\begin{array}{l}\text { MSTP for damageable } \\
\text { item }\end{array}$ & Linear & $\begin{array}{l}\text { Generalized reduced } \\
\text { gradient method }\end{array}$ \\
\hline $\begin{array}{l}\text { R a th and Dash } \\
{[11]}\end{array}$ & $\begin{array}{l}\text { Multi-objective linear } \\
\text { programming problem }\end{array}$ & $\begin{array}{l}\text { LInear, } \\
\text { hyperbolic }\end{array}$ & $\begin{array}{l}\text { Ranking function, } \\
\text { fuzzy programming }\end{array}$ \\
\hline $\begin{array}{l}\text { Rath and Das h } \\
{[12]}\end{array}$ & $\begin{array}{l}\text { Multi-objective linear } \\
\text { programming problem }\end{array}$ & $\begin{array}{l}\text { Linear, } \\
\text { exponential }\end{array}$ & $\begin{array}{l}\text { Ranking function, } \\
\text { fuzzy programming }\end{array}$ \\
\hline $\begin{array}{l}\text { V e r m a, B is w a l } \\
\text { and B is w a s [13] }\end{array}$ & $\begin{array}{l}\text { Multi-objective linear } \\
\text { transportation problem }\end{array}$ & $\begin{array}{l}\text { Hyperbolic, } \\
\text { exponential }\end{array}$ & Fuzzy programming \\
\hline
\end{tabular}

As seen from the literature, most of the papers deal with the standard constraint form of MSTP, but not the mixed constraint form. Moreover, linear membership functions have been used in almost all of these studies due to their computational 
efficiency. With these motivations, we used the hyperbolic membership function to present the membership degrees more precisely, and also focused on the mixed constraint form of MSTP which is a more general structure. Thus, a fuzzy programming method using the hyperbolic membership function is developed for solving MSTP with mixed constraints. A numerical example is given to compare the solutions of the proposed method with the linear and hyperbolic membership functions. Therefore, we can get a satisfactory solution to MSTP.

The organization of the paper is in five sections. In Section 2, the mathematical model of MSTP with mixed constraints is given. The next Section 3 presents the solution procedure of our problem using the hyperbolic membership function. In Section 4, an illustrative example is solved, and a comparison is presented for linear and hyperbolic membership functions. Finally, the conclusion is given in Section 5.

\section{MSTP with mixed constraints}

In real-life problems, the constraints of transportation problems are not generally in the same form. In some cases, the decision-maker may have specified a supply amount that must be provided from a particular source. Then, the corresponding supply constraint will be "greater than or equal to" form. Similarly, when the amount of resources owned by any supplier is limited (i.e., have an upper limit), then the corresponding supply constraint will be "less than or equal to" form. These different types of inequalities may also appear in any demand or conveyance constraints. With this motivation, MSTP with mixed constraints, which we focused on this paper, enables to model in a more realistic aspect.

MSTP with mixed constraints aims to find the minimal cost for transporting a product from $m$ supplies to $n$ demands through $K$ conveyances, where their capacities are: $a_{i}, i=1,2, \ldots, m ; b_{j}, j=1,2, \ldots, n ;$ and $e_{k}, k=1,2, \ldots, K$, respectively. And suppose that the cost of $p$-th objective function $Z_{p}(x)$, $p=1,2, \ldots, P$, is denoted by $c_{i j k}^{p}$ which corresponds to $x_{i j k}$. Then, the mathematical model of the MSTP with mixed constraints can be defined as

$$
\min Z_{p}(\mathbf{x})=\sum_{i=1}^{m} \sum_{j=1}^{n} \sum_{k=1}^{K} c_{i j k}^{p} x_{i j k}, \quad p=1,2, \ldots, P,
$$

s.t.

$$
\begin{gathered}
\sum_{j} \sum_{k} x_{i j k}=a_{i}, i \in I_{1} ; \quad \sum_{j} \sum_{k} x_{i j k} \geq a_{i}, i \in I_{2} ; \quad \sum_{j} \sum_{k} x_{i j k} \leq a_{i}, \quad i \in I_{3}, \\
\sum_{k} \sum_{i} x_{i j k}=b_{j}, \quad j \in J_{1} ; \sum_{k} \sum_{i} x_{i j k} \geq b_{j}, \quad j \in J_{2} ; \quad \sum_{k} \sum_{i} x_{i j k} \leq b_{j}, \quad j \in J_{3}, \\
\sum_{i} \sum_{j} x_{i j k}=e_{k}, \quad k \in K_{1} ; \quad \sum_{i} \sum_{j} x_{i j k} \geq e_{k}, k \in K_{2} ; \quad \sum_{i} \sum_{j} x_{i j k} \leq e_{k}, k \in K_{3}, \\
x_{i j k} \geq 0, \quad i \in I_{1} \cup I_{2} \cup I_{3}=I ; \quad j \in J_{1} \cup J_{2} \cup J_{3}=J ; \quad k \in K_{1} \cup K_{2} \cup K_{3}=K,
\end{gathered}
$$

where the subscripts on $Z_{p}(x)$ and $c_{i j k}^{p}$ denote the $p$-th objective function, and: $a_{i}>0, \forall i ; b_{j}>0, \forall j ; e_{k}>0, \forall k ; c_{i j k}^{p} \geq 0, \forall i, j, k, p$. Also, $I_{1}, I_{2}$, and $I_{3}$ 
refer to "equality", "greater than or eqaual to", and "less than or equal to" type of constraints, respectively. Similarly the partition on the index set of demands and conveyances can be defined.

Let the feasible region of (1) is denoted by $S$. It is assumed that $S$ is a convex and compact set.

Definition 1. $\mathbf{x}^{*} \in S$ is a Pareto-optimal (strongly-efficient) solution if there does not exist another $\mathbf{x} \in S$ such that $Z_{p}(\mathbf{x}) \leq Z_{p}\left(\mathbf{x}^{*}\right) \forall p$, and $Z_{p}(\mathbf{x}) \neq Z_{p}\left(\mathbf{x}^{*}\right)$ $\exists p ;$ where $\mathbf{x}^{*}=\left\{x_{i j k}\right\}$.

Definition 2. A feasible $\mathbf{x}^{*} \in S$ is a compromise solution of (1) iff $\mathbf{x}^{*} \in E$ and $Z_{p}\left(\mathbf{x}^{*}\right) \leq \wedge_{\mathbf{x} \in S} Z(\mathbf{x})$ where $Z(\mathbf{x})=\left(Z_{1}(\mathbf{x}), Z_{2}(\mathbf{x}), \ldots, Z_{p}(\mathbf{x})\right), \wedge$ stands for "minimum" and the set of Pareto-optimal solutions is $E$.

\section{A fuzzy programming approach to MSTP with mixed constraints}

3.1. Designing the linear membership functions of the objectives

The linear membership functions of each objective can be written in the following way:

$$
\mu_{P}\left(Z_{P}(\mathbf{x})\right)=\left\{\begin{array}{cl}
1, & Z_{P}<L_{p}, \\
\frac{U_{P}-Z_{p}(\mathbf{x})}{U_{p}-L_{p}}, & L_{p} \leq Z_{p} \leq U_{p}, \\
0, & Z_{p}>U_{p},
\end{array}\right.
$$

where $\max _{\mathbf{x} \in S} Z_{p}(\mathbf{x})=U_{p}$ and $\min _{\mathbf{x} \in S} Z_{p}(\mathbf{x})=L_{p}, \forall p=1, . ., P$.

By using Zimmermann's "min" fuzzy operator model, and introducing a new auxiliary variable $\lambda=\min \mu_{p}\left(Z_{p}\right)$, problem (1) can be converted to

$\max \lambda$,

$$
\text { s.t. } \quad \mu_{p}\left(Z_{p}\right) \geq \lambda, \quad p=1, \ldots, P, \mathbf{x} \in S .
$$

Problem (3) is the model that corresponds to Zimmermann's min operator. The $\lambda^{*}$ solution represents the common satisfactory level of all objectives. Here, the word "common" means the lowest level of satisfaction achieved for each objective of (1).

3.2. Designing the non-linear (hyperbolic) membership functions of the objectives

Although the linear membership function is widely utilized in many real-world decision-making problems, the usage of the non-linear membership function can provide a more realistic conclusion than the linear one in some practical applications. The rate of satisfaction of nonlinear membership functions is not always constant as in linear membership functions. The shape of the nonlinear membership function alters in accordance with an $\alpha$ parameter value which provides more flexibility in 
decision-making problems. Thus in this paper, we use the hyperbolic membership function instead of using a linear membership function.

The hyperbolic membership function of the $p$-th objective function can be determined as

(4) $\mu_{p}^{H}\left(Z_{p}(\mathbf{x})\right)=\left\{\begin{array}{ccc}1, & Z_{p} \leq L_{p}, \\ \frac{1}{2}+\frac{\left[e^{\left(\frac{L_{p}+U_{p}}{2}-Z_{p}(\mathbf{x}) \alpha_{p}\right.}-e^{-\left(\frac{L_{p}+U_{p}}{2}-Z_{p}(\mathbf{x}) \alpha_{p}\right.}\right]}{2\left[e^{\left(\frac{L_{p}+U_{p}}{2}-Z_{p}(\mathbf{x}) \alpha_{p}\right.}+e^{-\left(\frac{L_{p}+U_{p}}{2}-Z_{p}(\mathbf{x}) \alpha_{p}\right]}\right]} & L_{p}<Z_{p}<U_{p}, \\ 0, & Z_{p} \geq U_{p},\end{array}\right.$

$\alpha_{p}$ is named as the shape parameter. Using (4), the fuzzy programming method integrated with hyperbolic membership functions for the solution of (1) can be presented as

$\max \lambda$,

$$
\text { s.t. } \quad \mu_{p}^{\mathrm{H}}\left(Z_{p}(\mathbf{x})\right) \geq \lambda, \quad p=1, \ldots, P, \mathbf{x} \in S,
$$

where the hyperbolic membership function is given as

$$
\begin{gathered}
\mu_{p}^{\mathrm{H}}\left(Z_{p}(\mathbf{x})\right)=\frac{1}{2}+\frac{\left[e^{\left(\frac{L_{p}+U_{p}}{2}-Z_{p}(\mathbf{x}) \alpha_{p}\right.}-e^{-\left(\frac{L_{p}+U_{p}}{2}-Z_{p}(\mathbf{x}) \alpha_{p}\right.}\right]}{2\left[e^{\left(\frac{L_{p}+U_{p}}{2}-Z_{p}(\mathbf{x}) \alpha_{p}\right.}+e^{-\left(\frac{L_{p}+U_{p}}{2}-Z_{p}(\mathbf{x}) \alpha_{p}\right.}\right]}= \\
=\frac{1}{2}\left(1+\tanh \left(\left(\frac{L_{p}+U_{p}}{2}-Z_{p}(\mathbf{x})\right) \alpha_{p}\right)\right) .
\end{gathered}
$$

Considering (6), the problem (5) can be rewritten as follows:

$$
\text { s.t. } \lambda \leq \frac{1}{2} \tanh \left(\left(\frac{L_{p}+U_{p}}{2}-Z_{p}(\mathbf{x})\right) \alpha_{p}\right)+\frac{1}{2}, p=1,2, \ldots, P, \mathbf{x} \in S .
$$

Introducing a new variable $x_{\mathrm{H}}=\tanh ^{-1}(2 \lambda-1)$, the foregoing problem (7) becomes

$$
\max \lambda
$$

$$
\text { s.t. } x_{\mathrm{H}} \leq\left(\frac{L_{p}+U_{p}}{2}-Z_{p}(\mathbf{x})\right) \alpha_{p}, p=1, . ., P, \mathbf{x} \in S .
$$

Since $\lambda=\frac{1}{2} \tanh \left(x_{\mathrm{H}}\right)+\frac{1}{2}$ and tanh is a strictly increasing function, (8) can be converted to the following linear programming problem: 
(9)

$\max x_{\mathrm{H}}$,

$$
\text { s.t. } \alpha_{p} Z_{p}(\mathbf{x})+x_{\mathrm{H}} \leq \frac{L_{p}+U_{p}}{2} \alpha_{p}, \quad p=1, \ldots, P, \mathbf{x} \in S .
$$

After the optimal solution $\left(\mathbf{x}^{*}, \mathbf{x}_{\mathrm{H}}^{*}\right)$ is found by solving the problem (9), the common satisfactory level $\lambda^{*}$ and the Pareto-optimal solution to the problem (1) can be found by $\left(\lambda^{*}, \mathbf{x}^{*}\right)=\left(\frac{1}{2} \tanh \left(\mathbf{x}_{\mathrm{H}}^{*}\right)+\frac{1}{2}, \mathbf{x}^{*}\right)$.

\section{Illustrative numerical example}

Let us implement our solution procedure to following numerical example taken from [2].

Supplies: $a_{1}=8 ; a_{2}=9 ; a_{3}=5$;

Demands: $b_{1}=7 ; b_{2}=6 ; b_{3}=5$;

Conveyances capacities: $e_{1}=10 ; e_{2}=5 ; e_{3}=6$;

Penalties of the first objective: $c_{i j k}^{1}$

\begin{tabular}{|c|c|c|c|}
\hline$i$ & 1 & 2 & 3 \\
\hline 1 & 9 & 6 & 3 \\
\hline 2 & 5 & 9 & 6 \\
\hline 3 & 2 & 2 & 1 \\
\hline \multicolumn{4}{|c|}{ Conveyance $1(k=1)$} \\
\hline
\end{tabular}

\begin{tabular}{|c|c|c|c|}
\hline$i$ & 1 & 2 & 3 \\
\hline 1 & 12 & 9 & 7 \\
\hline 2 & 6 & 11 & 8 \\
\hline 3 & 2 & 7 & 9 \\
\hline \multicolumn{3}{|c|}{ Conveyance $2(k=2)$} \\
\hline
\end{tabular}

\begin{tabular}{|c|c|c|c|}
\hline$i$ & 1 & 2 & 3 \\
\hline 1 & 9 & 7 & 7 \\
\hline 2 & 5 & 3 & 6 \\
\hline 3 & 1 & 7 & 3 \\
\hline \multicolumn{3}{|c|}{ Conveyance 3 $(k=3)$} \\
\hline
\end{tabular}

Penalties of the second objective: $c_{i j k}^{2}$

\begin{tabular}{|c|c|c|c|}
\hline$i$ & 1 & 2 & 3 \\
\hline 1 & 2 & 1 & 9 \\
\hline 2 & 2 & 4 & 8 \\
\hline 3 & 5 & 8 & 5 \\
\hline \multicolumn{4}{|c|}{ Conveyance $1(k=1)$} \\
\hline
\end{tabular}

\begin{tabular}{|c|c|c|c|}
\hline$i$ & 1 & 2 & 3 \\
\hline 1 & 9 & 4 & 9 \\
\hline 2 & 8 & 5 & 6 \\
\hline 3 & 2 & 9 & 2 \\
\hline \multicolumn{4}{|c|}{ Conveyance 2 $(k=2)$} \\
\hline
\end{tabular}

\begin{tabular}{|c|c|c|c|}
\hline$i$ & 1 & 2 & 3 \\
\hline 1 & 8 & 1 & 5 \\
\hline 2 & 1 & 2 & 9 \\
\hline 3 & 7 & 7 & 5 \\
\hline \multicolumn{4}{|c|}{ Conveyance 3 $(k=3)$} \\
\hline
\end{tabular}

Penalties of the second objective: $c_{i j k}^{3}$

\begin{tabular}{|c|c|c|c|}
\hline$i$ & 1 & 2 & 3 \\
\hline 1 & 2 & 3 & 8 \\
\hline 2 & 2 & 5 & 9 \\
\hline 3 & 1 & 8 & 5 \\
\hline \multicolumn{4}{|c|}{ Conveyance $1(k=1)$} \\
\hline
\end{tabular}

\begin{tabular}{|c|c|c|c|}
\hline$i$ & 1 & 2 & 3 \\
\hline 1 & 4 & 6 & 4 \\
\hline 2 & 5 & 6 & 6 \\
\hline 3 & 9 & 3 & 7 \\
\hline \multicolumn{4}{|c|}{ Conveyance 2 $(k=2)$} \\
\hline
\end{tabular}

\begin{tabular}{|c|c|c|c|}
\hline$i$ & 1 & 2 & 3 \\
\hline 1 & 6 & 4 & 9 \\
\hline 2 & 3 & 6 & 3 \\
\hline 3 & 1 & 9 & 11 \\
\hline \multicolumn{4}{|c|}{ Conveyance 3 $(k=3)$} \\
\hline
\end{tabular}

Then, the corresponding MSTP can be written explicitly as follows: 

$\min Z_{p}(\mathbf{x})=\sum_{i=1}^{3} \sum_{j=1}^{3} \sum_{k=1}^{3} c_{i j k}^{p} x_{i j k}, \quad p=1,2,3$,

$$
\begin{gathered}
\text { s.t. } \sum_{j=1}^{3} \sum_{k=1}^{3} x_{1 j k}=8, \sum_{j=1}^{3} \sum_{k=1}^{3} x_{2 j k} \geq 9, \sum_{j=1}^{3} \sum_{k=1}^{3} x_{3 j k} \leq 5, \sum_{k=1}^{3} \sum_{i=1}^{3} x_{i 1 k}=7, \\
\sum_{k=1}^{3} \sum_{i=1}^{3} x_{i 2 k} \geq 6, \sum_{k=1}^{3} \sum_{i=1}^{3} x_{i 3 k} \leq 5, \sum_{i=1}^{3} \sum_{j=1}^{3} x_{i j 1}=10, \sum_{i=1}^{3} \sum_{j=1}^{3} x_{i j 2} \geq 5, \sum_{i=1}^{3} \sum_{j=1}^{3} x_{i j 3} \leq 6 .
\end{gathered}
$$

Let the feasible region of (10) is denoted by $\hat{S}$.

Optimizing the objective functions of (10) individually, the aspired and the highest acceptable level of achievement for objective functions are found as: $L_{1}=75, U_{1}=133 ; L_{2}=32, U_{2}=80 ; L_{3}=53.5, U_{3}=130$. Then, by using (2), the linear membership functions are constructed as:

$$
\mu_{1}\left(Z_{1}(\mathbf{x})\right)=\frac{133-Z_{1}(\mathbf{x})}{58}, \mu_{2}\left(Z_{2}(\mathbf{x})\right)=\frac{80-Z_{2}(\mathbf{x})}{48}, \mu_{3}\left(Z_{3}(\mathbf{x})\right)=\frac{130-Z_{3}(\mathbf{x})}{76.5} .
$$

\subsection{Solving MSTP using hyperbolic membership function}

Using (6), the hyperbolic membership function of $Z_{1}$ can be constructed easily. For this objective, Fig. 1 presents the graph for the linear and hyperbolic membership functions. Fig. 1 shows that the hyperbolic membership function gives better membership values for the satisfaction degrees above the intersection point. Similarly, the graphs of the remaining objective functions can be drawn as well.

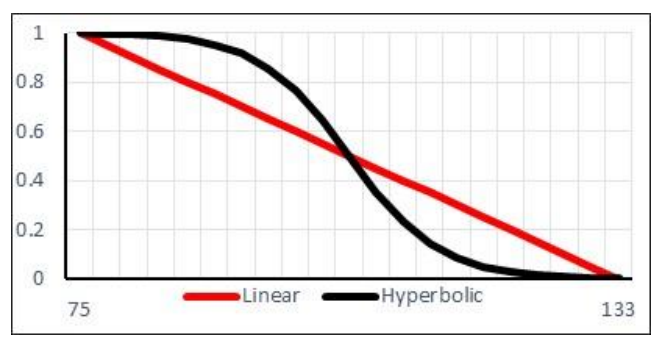

Fig. 1. The linear and hyperbolic membership functions of $Z_{1}$

Then, the problem corresponds to (9) and can be rewritten as:

$$
\begin{gathered}
\max x_{\mathrm{H}}, \\
\text { s.t. } 0.103 Z_{1}(\mathbf{x})-x_{\mathrm{H}} \geq 10.35,0.125 Z_{2}(\mathbf{x})-x_{\mathrm{H}} \geq 7.28, \\
0.078 Z_{3}(\mathbf{x})-x_{\mathrm{H}} \geq 7.34, \quad \mathbf{x} \in \hat{S} .
\end{gathered}
$$

where $\alpha_{1}=0.103, \alpha_{2}=0.125, \alpha_{3}=0.078$. We note here that the shape parameters are determined by the formula $\alpha_{p}=6 /\left(U_{p}-L_{p}\right), p=1,2,3$, which is generally used in the literature. To offer more Pareto-optimal solutions to the decision-maker, it is possible to obtain various solutions by choosing different shape parameters. Solving $(11), \mathbf{x}_{1}^{*}$ and $x_{\mathrm{H}}^{*}$ is obtained as follows: 


$$
\begin{aligned}
& x_{\mathrm{H}}^{*}=1.58 \text {, }
\end{aligned}
$$

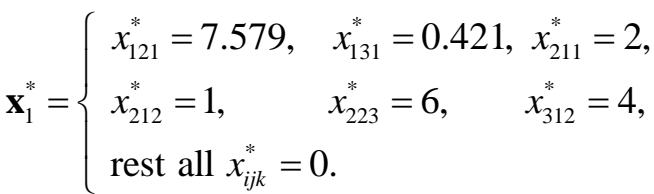

Using the equation $\lambda_{1}^{*}=\frac{1}{2} \tanh \left(\mathbf{x}_{\mathrm{H}}^{*}\right)+\frac{1}{2}$, the common satisfactory level of membership functions is found as $\lambda_{1}^{*}=0.96$. For $\mathbf{x}_{1}^{*}$, the objective function values are $Z_{1}\left(\mathbf{x}_{1}^{*}\right)=88.74, Z_{2}\left(\mathbf{x}_{1}^{*}\right)=43.37, Z_{3}\left(\mathbf{x}_{1}^{*}\right)=107.11$.

\subsection{Solving MSTP using linear membership function}

In this subsection, to show the effectiveness of the hyperbolic membership function, the Pareto-optimal solution will be obtained by the widely used linear membership function (2), and a comparison will be presented.

The problem corresponding to (3) can be written as follows:

$$
\max \lambda_{2} \text {, }
$$

$$
\begin{gathered}
\text { s.t. } \mu_{1}\left(Z_{1}(\mathbf{x})\right)=\frac{133-Z_{1}(\mathbf{x})}{58} \geq \lambda_{2}, \mu_{2}\left(Z_{2}(\mathbf{x})\right)=\frac{80-Z_{2}(\mathbf{x})}{48} \geq \lambda_{2}, \\
\mu_{3}\left(Z_{3}(\mathbf{x})\right)=\frac{130-Z_{3}(\mathbf{x})}{76.5} \geq \lambda_{2}, \mathbf{x} \in \hat{S} .
\end{gathered}
$$

Solving (12), $\mathbf{x}_{2}^{*}$ and $\lambda_{2}^{*}$ are obtained as

$$
\mathbf{x}_{2}^{*}=\left\{\begin{array}{c}
x_{121}^{*}=7.17, \quad x_{122}^{*}=0.830, x_{211}^{*}=2.83, \\
x_{212}^{*}=2.779, x_{223}^{*}=3.391, x_{312}^{*}=1.391, \\
\text { rest all } x_{i j k}^{*}=0, \\
\lambda_{2}^{*}=0.67 .
\end{array}\right.
$$

For $\mathbf{x}_{2}^{*}$, the objective function values are $Z_{1}\left(\mathbf{x}_{2}^{*}\right)=94.27, Z_{2}\left(\mathbf{x}_{2}^{*}\right)=47.95$, $Z_{3}\left(\mathbf{x}_{2}^{*}\right)=78.91$. The satisfaction degrees of all objective functions are summarized in Table 2.

Table 2. The comparison of membership functions

\begin{tabular}{|c|c|c|c|c|c|c|c|}
\hline \multirow{2}{*}{ Value } & \multicolumn{2}{|c|}{ Single-objective case } & \multicolumn{4}{|c|}{ Multi-objective case } \\
\cline { 2 - 8 } & $Z_{1}$ & $Z_{2}$ & $Z_{3}$ & $Z_{p}\left(\mathbf{x}_{1}^{*}\right)$ & $\mu_{p}^{\mathrm{H}}\left(Z_{p}\left(\mathbf{x}_{1}^{*}\right)\right)$ & $Z_{p}\left(\mathbf{x}_{2}^{*}\right)$ & $\mu_{p}\left(Z_{p}\left(\mathbf{x}_{2}^{*}\right)\right)$ \\
\hline$Z_{1}$ & 75 & 133 & 106 & 88.74 & 0.96 & 94.27 & 0.67 \\
\hline$Z_{2}$ & 80 & 32 & 60.5 & 43.37 & 0.83 & 47.95 & 0.67 \\
\hline$Z_{3}$ & 130 & 83 & 53.5 & 107.11 & 0.5 & 78.91 & 0.67 \\
\hline
\end{tabular}


In the fuzzy approach used the linear membership function, both objective functions are satisfied at the same satisfactory level. In our proposed approach, in which the hyperbolic membership function is used, the $Z_{1}$ and $Z_{2}$ objectives have reached a very high level of satisfaction compared to the linear one. In particular, the $Z_{1}$ objective function is really close to the full satisfaction level. On the other hand, a slight decrease was observed in the satisfaction level of the $Z_{3}$ in our proposed approach. While both solutions obtained with linear and hyperbolic membership functions are Pareto-optimal, our proposed approach gives a better average satisfaction value of 0.76 as can be seen from Fig. 2, the linear one gives an average satisfaction value of 0.67 . Table 2 also presents the objective function values of single-objective problems in which each $Z_{p}, \quad p=1,2,3$, is optimized with three different sets of penalties under the same constraints. As can be seen from these values, although the solutions of the multi-objective problems cannot reach all the individual optimal values, these solutions fulfill all the objectives at a common satisfaction degree.

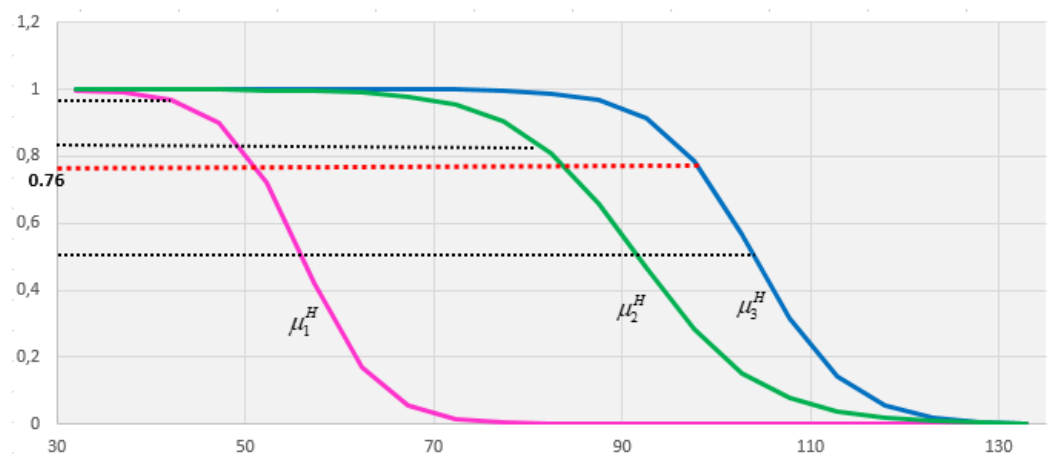

Fig. 2. The hyperbolic membership functions of all objectives and satisfaction levels

\section{Conclusion}

When decision-makers try to optimize conflicting goals in multi-objective problems and some available information is uncertain, their task is very difficult. Zimmermann's fuzzy approach, which uses fuzzy set theory developed to deal with such uncertain situations, is one of the most widely used methods in the literature. In Zimmermann's approach, the linear membership function is used to scale the objective functions. However, the non-linear membership function is much more versatile than linear types and can generate better results for objective functions' satisfactory levels. Therefore, a hyperbolic membership function is used instead of a linear membership function for solving the mixed constraint form of MSTP which is a more general structure. The advantages of using the hyperbolic membership function can be pointed out in two aspects. Firstly, when the Pareto-optimal solutions obtained by fuzzy approaches using hyperbolic and linear membership functions are compared, it can be stated that the hyperbolic approach achieves a better satisfaction level on average. Secondly, the usage of the hyperbolic membership function is more 
realistic than the linear one in some practical applications. That is, the decision-maker can opt for the membership function, which is presenting a better solution for the objectives being of higher priority.

\section{References}

1. A nuradha, D., M. J a y 1 a k shmi, G. Deepa, V. S u j a tha. Solution of Multiobjective Solid Transportation Problem in Fuzzy Approach. - AIP Conference, Vol. 2177, 2019, No 1, pp. 5-5.

2. B i t, A. K., M. P. B i s w a 1, S. S. A 1 a m. Fuzzy Programming Approach to Multiobjective Solid Transportation Problem. - Fuzzy Sets and Systems, Vol. 57, 1993, pp. 183-194.

3. B it, A. K. Fuzzy Programming with Hyperbolic Membership Functions for Multiobjective Capacitated Transportation Problem. - Operational Research Society of India, Vol. 41, 2004, pp. $106-120$

4. B it, A. K. Fuzzy Programming with Hyperbolic Membership Functions for Multi-Objective Capacitated Solid Transportation Problem. - The Journal of Fuzzy Mathematics, Vol. 13, 2005, pp. 373-385.

5. B o d k h e, S. G., V.H. B a j a j, D. B. D h a i g u d e. Fuzzy Programming Technique to Solve MultiObjective Solid Transportation Problem with Some Non-Linear Membership Functions. Advances in Computational Research, Vol. 2, 2010, No 1, pp. 15-20.

6. Che n, L., J. Peng, B. Zhang. Uncertain Goal Programming Models for Bicriteria Solid Transportation Problem. - Applied Soft Computing, Vol. 51, 2017, pp. 49-59.

7. L e b e r ling, H. On Finding Compromise Solutions in Multicriteria Problems Using the Fuzzy Min-Operator. - Fuzzy Sets and Systems, Vol. 6, 1981, No 2, pp. 105-118.

8. P e i d r o, D., P. V a s a n t. Transportation Planning with Modified S-Curve Membership Functions Using an Interactive Fuzzy Multi-Objective Approach. - Applied Soft Computing, Vol. 11, 2011, No 2, pp. 2656-2663.

9. P e n e v a, V., I. P o p c h e v. Fuzzy Multicriteria Decision Making. - Cybernetics and Information Technologies, Vol. 2, 2002, No 1, pp. 1-26.

10. Pram anik, S., D. K. Jan a, M. Maiti. Multi-Objective Solid Transportation Problem in Imprecise Environments. - Journal of Transportation Security, Vol. 6, 2013, No 2, pp. 131-150.

11. R a th, P., R. B. D a s h. Solution of Fuzzy Multi-Objective Linear Programming Problems Using Fuzzy Programming Techniques Based on Hyperbolic Membership Functions. - Journal of Computer and Mathematical Sciences, Vol. 7, 2016, No 12, pp. 653-662.

12. R a th, P., R. B. D a s h. Solution of Fuzzy Multi-Objective Linear Programming Problems Using Fuzzy Programming Techniques Based on Exponential Membership Functions. - Inter J. Mathematics Trends and Tech, Vol 41, 2017, pp. 289-292.

13. Verma, R., M. P. B is w a 1, A. B is w as. Fuzzy Programming Technique to Solve MultiObjective Transportation Problems with Some Non-Linear Membership Functions. - Fuzzy Sets and Systems, Vol. 91, 1997, No 1, pp. 37-43.

Received: 23.09.2021; Second Version: 02.11.2021; Accepted: 12.11.2021 